\title{
Ratios of congruent numbers
}

\author{
by \\ Ashvin Rajan (Baltimore, MD) and \\ FranÇOIS Ramaroson (Washington, DC)
}

1. Introduction. A rational right triangle is a right triangle whose sides are all positive rational numbers. Such a triangle is denoted $\{a, b, c\}$ where $a$ and $b$ are the legs, and $c$ is the hypotenuse. Throughout this paper, a squarefree integer is understood to be a positive integer which is not divisible by the square of an integer greater than 1. A congruent number is a square-free integer which is the area of a rational right triangle. A square-free integer $N$ is a congruent number if and only if the elliptic curve $N y^{2}=\left(x^{2}-1\right) x$ has positive rank. For details, see Koblitz [7].

In the spirit of Euclid's proof of the infinitude of prime numbers, one can also show that there are infinitely many congruent numbers as follows: If there were only finitely many of them, say $N_{1}, \ldots, N_{r}$, all greater than 1 , then consider $N=N_{1} \cdots N_{r}$. Elementary number theory shows that $\operatorname{sqf}\left(N^{3}-N\right)$, the square-free part of $N^{3}-N$, cannot be 1 . Moreover, it is a congruent number which cannot be any of the $N_{i}$ 's. Indeed, if it were $N_{1}$, say, let $M=N_{2} \cdots N_{r}$ and $d=\operatorname{gcd}\left(N_{1}, M\right)$. Writing $N_{1}=d n$ and $M=d m$ with $\operatorname{gcd}(m, n)=1$, one sees that $\operatorname{sqf}(m) \operatorname{sqf}\left(N_{1}^{2} M^{2}-1\right)=d$. This last equality implies that $\operatorname{sqf}\left(N_{1}^{2} M^{2}-1\right)$ divides $d$, and hence $M$, but at the same time, since it divides $N_{1}^{2} M^{2}-1$, it must be 1 , and this is impossible.

Chahal [2] established that the residue classes of 1,2,3,5,6,7 modulo 8 contain infinitely many congruent numbers. Bennett [1] extended Chahal's result by showing that if $a$ and $m$ are positive integers such that $\operatorname{gcd}(a, m)$ is square-free, then the residue class of $a$ modulo $m$ contains infinitely many congruent numbers.

In this paper we prove the following:

2000 Mathematics Subject Classification: Primary 11G05; Secondary 14 G05.

The authors wish to thank J. S. Chahal and the referee for useful suggestions. 
MAIN TheOREm. If $k$ and $l$ are positive, square-free, coprime integers, then there exist infinitely many pairs $(M, N)$ of congruent numbers such that $l N=k M$.

COROLlary. If $k$ and $l$ are positive, square-free, coprime integers, then there exist infinitely many square-free integers $N$ such that both $k N$ and $l N$ are congruent numbers.

2. Holm's curve and its jacobian. We consider the following nonsingular curve of genus one:

$$
H: \quad l x\left(x^{2}-1\right)=k y\left(y^{2}-1\right),
$$

where $k$ and $l$ are coprime, square-free integers. In a slightly different form, this curve was considered by Holm [5], in his work on right triangles whose areas are in a given ratio. The jacobian of $H$ is the elliptic curve

$$
E: \quad Y^{2}=X^{3}-3 k^{2} l^{2} X+k^{2} l^{2}\left(k^{2}+l^{2}\right) .
$$

The following proposition is easily proved.

Proposition 2.1.

(i) The discriminant of $E$ is $-3^{3} k^{4} l^{4}\left(k^{2}-l^{2}\right)^{2}$.

(ii) The $j$-invariant of $E$ is $-2^{8} 3^{3} k^{2} l^{2} /\left(k^{2}-l^{2}\right)^{2}$.

(iii) The following integral points lie on $E$ :

$(-k l, \pm k l(k+l)),\left(k^{2}, \pm k\left(k^{2}-l^{2}\right)\right),(k l, \pm k l(k-l)),\left(l^{2}, \pm l\left(k^{2}-l^{2}\right)\right)$.

(iv) $E$ has positive rank, since $\left(l^{2}, l\left(k^{2}-l^{2}\right)\right)$ is a point of infinite order.

(v) The rational transformations relating $H$ and $E$ are

$$
\begin{aligned}
x=\frac{k\left(X-l^{2}\right)}{Y}, & y=\frac{l\left(X-k^{2}\right)}{Y}, \\
X=\frac{k l(k x-l y)}{l x-k y}, & Y=\frac{k l\left(k^{2}-l^{2}\right)}{l x-k y} .
\end{aligned}
$$

Let $A_{x}=x\left(x^{2}-1\right)$ and $A_{y}=y\left(y^{2}-1\right)$. Then every rational point $(x, y)$ on $H$, that is, not in the set

$$
\{(0,0),( \pm 1, \pm 1),( \pm 1,0),(0, \pm 1)\},
$$

gives rise to two rational right triangles whose areas are in the ratio

$$
\frac{A_{x}}{A_{y}}=\frac{k}{l} .
$$

Indeed, if both $A_{x}$ and $A_{y}$ are positive, the rational right triangles $\left\{x^{2}-1\right.$, $\left.2 x, x^{2}+1\right\}$ (for $x>0$ ), or $\left\{1-x^{2},-2 x, x^{2}+1\right\}$ (for $x<0$ ), will have area $A_{x}$, and similarly for $A_{y}$, while if $A_{x}$ and $A_{y}$ are both negative, the rational right triangles $\left\{x^{2}-1,-2 x, x^{2}+1\right\}$ (for $x>0$ ), or $\left\{1-x^{2}, 2 x, x^{2}+1\right\}$ (for $x<0$ ), will have area $-A_{x}$, and similarly for $-A_{y}$. Therefore, every rational point 
$(x, y)$ on $H$ which is not in the set mentioned above produces a pair of congruent numbers, $\left(N_{x}, N_{y}\right)$, when we take the square-free parts $N_{x}$ of $A_{x}$ and $N_{y}$ of $A_{y}$ respectively.

If we choose a rational point $(X, Y)$ in $E(\mathbb{Q})$ different from those listed in Proposition 2.1(iii) and employ the transformations, we get "areas" $A_{x}(X, Y)$ and $A_{y}(X, Y)$. We will show that there are infinitely many points $(X, Y)$ in $E(\mathbb{Q})$ for which $l$ is prime to the square-free part of $A_{x}(X, Y)$ and $k$ is prime to the square-free part of $A_{y}(X, Y)$. In order to do this, we will use well-known properties of $p$-adic filtrations.

3. The $p$-adic filtration on global points. Let $E$ be an elliptic curve given as a Weierstrass model with coefficients in $\mathbb{Z}$, and $p$ a prime at which the model is minimal. We then have the $p$-adic filtration

$$
E\left(\mathbb{Q}_{p}\right) \supset E_{0}\left(\mathbb{Q}_{p}\right) \supset E_{1}\left(\mathbb{Q}_{p}\right) \supset E_{2}\left(\mathbb{Q}_{p}\right) \supset \cdots
$$

The following facts on $p$-adic filtrations are well-known (see Knapp [6] or Silverman [8], for instance).

(1) $E_{0}\left(\mathbb{Q}_{p}\right)$ is the set of points whose reduction $\bmod p$ is non-singular.

(2) $E_{1}\left(\mathbb{Q}_{p}\right)$ is the kernel of reduction $\bmod p$.

(3) $E\left(\mathbb{Q}_{p}\right) / E_{0}\left(\mathbb{Q}_{p}\right)$ and $E_{0}\left(\mathbb{Q}_{p}\right) / E_{1}\left(\mathbb{Q}_{p}\right)$ are finite groups.

(4) For each $n \geq 1, E_{n}\left(\mathbb{Q}_{p}\right)=\left\{P \mid \operatorname{ord}_{p}(x(P)) \leq-2 n\right\}$.

(5) For each $n \geq 1, E_{n}\left(\mathbb{Q}_{p}\right) / E_{n+1}\left(\mathbb{Q}_{p}\right) \cong \mathbb{F}_{p}$.

Let

$$
E_{n}(\mathbb{Q})=E_{n}\left(\mathbb{Q}_{p}\right) \cap E(\mathbb{Q}) \quad \text { for each } n \geq 1 .
$$

The proofs of the following propositions are well-known.

Proposition 3.1. For each $m \geq 1$, if $E_{m}(\mathbb{Q}) / E_{m+1}(\mathbb{Q}) \cong \mathbb{F}_{p}$, then

$$
E_{m+1}(\mathbb{Q}) / E_{m+2}(\mathbb{Q}) \cong \mathbb{F}_{p}
$$

More generally, for any $n \geq m$, and for any $P \in E_{m}(\mathbb{Q})-E_{m+1}(\mathbb{Q})$,

$$
p^{n-m} P \in E_{n}(\mathbb{Q})-E_{n+1}(\mathbb{Q}) .
$$

Proposition 3.2. If $E$ has positive rank over $\mathbb{Q}$, then there is an integer $N \geq 1$ such that $E_{n}(\mathbb{Q}) / E_{n+1}(\mathbb{Q}) \cong \mathbb{F}_{p}$ for all $n \geq N$.

Next, we investigate the relationship between the filtrations on global points for a set of primes at each of which the model is minimal.

Let $S=\left\{p_{1}, \ldots, p_{s}\right\}$ be a set of distinct primes such that $E$ is minimal at each $p_{i}$. For each prime $p_{i}$, there is a $p_{i}$-adic filtration

$$
E\left(\mathbb{Q}_{p_{i}}\right) \supset E_{0}\left(\mathbb{Q}_{p_{i}}\right) \supset E_{1}\left(\mathbb{Q}_{p_{i}}\right) \supset \cdots .
$$


As before, we put $E_{n, p_{i}}(\mathbb{Q})=E_{n}\left(\mathbb{Q}_{p_{i}}\right) \cap E(\mathbb{Q})$. Proposition 3.2 implies that for each $i, 1 \leq i \leq s$, there exists an integer $N_{i}$ such that

$$
E_{n, p_{i}}(\mathbb{Q}) / E_{n+1, p_{i}}(\mathbb{Q}) \cong \mathbb{F}_{p_{i}} \text { for all } n \geq N_{i}
$$

Let $N=\max \left\{N_{1}, \ldots, N_{s}\right\}$, and for each $n \geq N$, let

$$
U_{n}=\bigcap_{i=1}^{s}\left(E_{n, p_{i}}(\mathbb{Q})-E_{n+1, p_{i}}(\mathbb{Q})\right) .
$$

Although somewhat lengthy, the proof of the following proposition is straightforward.

Proposition 3.3. If $E$ has positive rank over $\mathbb{Q}$, then there exists an integer $m \geq N$ such that $U_{m} \neq \emptyset$.

CoROllary 3.4. If $E$ has positive rank over $\mathbb{Q}$, then there exists an integer $m_{0}$ such that for all $m \geq m_{0}, U_{m} \neq \emptyset$.

4. Applications to the elliptic curve $E$. We now apply the general results of the previous section to the curve

$$
E: \quad Y^{2}=X^{3}-3 k^{2} l^{2} X+k^{2} l^{2}\left(k^{2}+l^{2}\right),
$$

which has positive rank over $\mathbb{Q}$. Recall that $k$ and $l$ are square-free, coprime positive integers.

Proposition 4.1. There exists an integer $n$ and an infinite set $\mathcal{P}$ of rational points in $E(\mathbb{Q})$ such that if $(X, Y) \in \mathcal{P}$ then, for any prime divisor $q$ of $l$ and for any prime divisor $p$ of $k$,

$$
\operatorname{ord}_{q}(X)=\operatorname{ord}_{p}(X)=-4 n, \quad \operatorname{ord}_{q}(Y)=\operatorname{ord}_{p}(Y)=-6 n .
$$

Proof. In the notation of the previous section, let $S$ be the set of all prime divisors of $k$ and $l$. By Proposition 2.1(i), and the assumptions on $k$ and $l, E$ is minimal at all primes in $S$. Applying Corollary 3.4, we find an integer $n$ such that $U_{2 n} \neq \emptyset$. Let $P \in U_{2 n}$, and consider the set of points $\mathcal{P}=\left\{P_{a}=r^{a} P \mid a \in \mathbb{N}\right\}$. Since $r \notin S$,

$$
\mathcal{P} \subset U_{2 n} \subset E(\mathbb{Q}) .
$$

Moreover, $\mathcal{P}$ is infinite since $P$ is of infinite order. The conclusions about the orders directly follow from the definition of $U_{2 n}$.

For each point $(X, Y) \in \mathcal{P}$, we form the "areas" $A_{x}=x\left(x^{2}-1\right)$ and $A_{y}=y\left(y^{2}-1\right)$ where

$$
x=\frac{k\left(X-l^{2}\right)}{Y}, \quad y=\frac{l\left(X-k^{2}\right)}{Y} .
$$

Theorem 4.2. For each $(X, Y) \in \mathcal{P}$, let $N_{x}$ (resp. $\left.N_{y}\right)$ be the square-free part of $A_{x}\left(\right.$ resp. $\left.A_{y}\right)$. Then

$$
l N_{x}=k N_{y}
$$


Proof. Proposition 4.1 implies that $\left(l, N_{x}\right)=\left(k, N_{y}\right)=1$. Since the point $(X, Y)$ is on $E$, the point $(x, y)$ is on $H$, and hence $l A_{x}=k A_{y}$. Taking the square-free parts of both sides yields the result.

Theorem 4.2 associates to every point $(X, Y)$ in the set $\mathcal{P}$ a pair of square-free integers $\left(N_{x}, N_{y}\right)$. We next establish that there are infinitely many such pairs $\left(N_{x}, N_{y}\right)$ associated to the infinite set $\mathcal{P}$. It is clear that if there were only a finite number of $N_{x}$, there would also be only a finite number of $N_{y}$, and vice versa.

Theorem 4.3. Associated with the infinite set of points $(X, Y)$ in $\mathcal{P}$, there are infinitely many pairs of square-free integers $\left(N_{x}, N_{y}\right)$.

Proof. Assume that there are only finitely many such pairs. Then there must exist a pair $(N, M)$ of square-free integers which is associated with infinitely many rational points $(X, Y)$ in $\mathcal{P}$. Using $(x, y)$ instead of $(X, Y)$, we find that in an $x y z w$-space, the algebraic variety

$$
(\mathcal{C}):\left\{\begin{array}{l}
l x\left(x^{2}-1\right)=k y\left(y^{2}-1\right), \\
x\left(x^{2}-1\right)=N z^{2} \\
y\left(y^{2}-1\right)=M w^{2}
\end{array}\right.
$$

is a non-singular algebraic curve, defined over $\mathbb{Q}$, having infinitely many rational points.

Lemma 4.4. In the xyz-space, the curve

$$
\left(\mathcal{C}_{1}\right):\left\{\begin{array}{l}
l x\left(x^{2}-1\right)=k y\left(y^{2}-1\right), \\
x\left(x^{2}-1\right)=N z^{2}
\end{array}\right.
$$

has only finitely many rational points.

Proof. In the projective space $P^{3}(\overline{\mathbb{Q}})$, with $x, y, z, t$ coordinates, the curve $\left(\mathcal{C}_{1}\right)$ has equations

$$
\left\{\begin{array}{l}
l x\left(x^{2}-t^{2}\right)=k y\left(y^{2}-t^{2}\right), \\
x\left(x^{2}-t^{2}\right)=N z^{2} t .
\end{array}\right.
$$

Let $\left(\mathcal{C}_{2}\right)$ be the elliptic curve $x\left(x^{2}-t^{2}\right)=N z t^{2}$, and consider the projection along $y$

$$
\left(\mathcal{C}_{1}\right) \rightarrow\left(\mathcal{C}_{2}\right), \quad(x, y, z, t) \mapsto(x, z, t) .
$$

This is a finite morphism of curves, of degree 3 , which is ramified over the point $(x, z, t)=(0,1,0)$. If we let $g\left(\left(\mathcal{C}_{1}\right)\right)$ be the genus of $\left(\mathcal{C}_{1}\right)$, the Hurwitz formula implies that $g\left(\left(\mathcal{C}_{1}\right)\right)>1$. Faltings' theorem $([3])$ now implies that $\left(\mathcal{C}_{1}\right)$ only has a finite number of rational points.

Note. One could also work out Exercise 7.2(d) in Hartshorne [4]. 
To finish the proof of Theorem 4.3, we observe that the projection from the curve $(\mathcal{C})$ to the curve $\left(\mathcal{C}_{1}\right)$ along $w$ is a rational map, defined over $\mathbb{Q}$, between curves, and is of degree 2 . Since $\left(\mathcal{C}_{1}\right)$ only has a finite set of rational points, so does $(\mathcal{C})$. This contradiction ends the proof of Theorem 4.3.

THEOREM 4.5. If $k$ and $l$ are positive, square-free, coprime integers, then there exist infinitely many pairs $(N, M)$ of congruent numbers such that $l N=k M$.

Proof. Consider the elliptic curve $E: Y^{2}=X^{3}-3 k^{2} l^{2} X+k^{2} l^{2}\left(k^{2}+l^{2}\right)$, the infinite set of rational points $\mathcal{P} \subset E(\mathbb{Q})$, and apply Theorems 4.2 and 4.3 .

The case $l=1$ is worth pointing out.

COROLlary 4.6. Given a positive, square-free integer $k$, there exist infinitely many pairs $(N, M)$ of congruent numbers such that $N=k M$.

\section{References}

[1] M. A. Bennett, Lucas' square pyramid problem revisited, Acta Arith. 105 (2002), 341-347.

[2] J. S. Chahal, On an identity of Desboves, Proc. Japan Acad. Ser. A Math. Sci. 60 (1984), 105-108.

[3] G. Faltings, Endlichkeitssätze für abelsche Varietäten über Zahlkörpern, Invent. Math. 73 (1983), 349-366.

[4] R. Hartshorne, Algebraic Geometry, Grad. Texts in Math. 52, Springer, New York, 1977.

[5] A. Holm, Some points in Diophantine analysis, Proc. Edinburgh Math. Soc. 22 (1903), 40-48.

[6] A. W. Knapp, Elliptic Curves, Math. Notes 40, Princeton Univ. Press, Princeton, NJ, 1992.

[7] N. Koblitz, Introduction to Elliptic Curves and Modular Forms, Grad. Texts in Math. 97, Springer, New York, 1984.

[8] J. H. Silverman, The Arithmetic of Elliptic Curves, Grad. Texts in Math. 106, Springer, New York, 1986.

3935 Cloverhill Road

Baltimore, MD 21218, U.S.A.

E-mail: AshvinRj@aol.com
Department of Mathematics

Howard University

Washington, DC 20059, U.S.A. E-mail: framaroson@howard.edu

Received on 20.5.2005

and in revised form on 21.10.2006 